\title{
La cyclosporine induit des rémissions du diabète insulino-dépendant
}

\author{
Les nouvelles \\ de ce numéro \\ ont été préparées par : \\ J.-P. Grünfeld \\ A. Kahn \\ J.-C. Dreyfus
}

1. Feutren G, Papoz L, Assan R, et al., Cyclosporin increases the rate and length of remissions in insulin-dependent diabetes of recent onset. Results of a multicentre double-blind trial. Lancet 1986; ii : 1 19-24.

2. Stiller CR, Dupre J, Gent M, et al. Effects of cyclosporine immunosuppression in insulindependent diabetes mellitus of recent onset. Science $1984 ; 223: 1363-7$.

3. Assan R, Feutren G, Debray-Sachs M, et al. Metabolic and immunological effects of cyclosporin in recently diagnosed type $I$ diabetes mellitus. Lancet $1985 ; \mathrm{i}: 67-7 \mathrm{r}$.

- Non-obese diabétique. *. Bio Breeding.
Des arguments convergents indiquent que le diabète de type I insulinodépendant (le diabète juvénile) est dû à la destruction des cellules $\beta$ des îlots de Langerhans pancréatiques par une réaction autoimmune. Plus précisément, il semble que les cellules $\mathrm{T}$ jouent le premier rôle par une action directe cytotoxique sur les cellules $\beta$. La cyclosporine est un immunosuppresseur puissant, sélectif des cellules T. Il était logique d'en éprouver les effets thérapeutiques dans le diabète de type I, d'autant qu'elle s'était révélée capable de prévenir le diabète de la souris $\mathrm{NOD}^{*}$ et du rat $\mathrm{BB}^{* *}$, deux modèles expérimentaux de diabète insulino-dépendant très proches du diabète humain.

Cent vingt-deux diabétiques au début de leur maladie (moins de six mois après l'apparition des premières manifestations cliniques, moins de deux mois après le début de l'insulinothérapie) ont été traités par la cyclosporine à la dose d'attaque de $7,5 \mathrm{mg} / \mathrm{kg} / \mathrm{j}$ ou par une préparation placebo en tous points identiques au produit actif. L'évolution sous traitement fut appréciée en double insu intégral, tant dans le suivi quotidien des malades que dans l'évaluation des résultats. Les rémissions complètes furent définies par la disparition complète du besoin d'insuline sans hypoglycémiant oral avec un bon équilibre métabolique jugé sur le taux d'hémoglobine glycosylée $(<6,5 \%)$. A neuf mois, après que les rémissions observées sous placebo aient cédé, le pourcentage de rémission complète était de $24 \%$ (contre $6 \%$ dans le groupe placebo, $\mathrm{p}<0, \mathrm{oI}$ ). La différence était encore plus nette si l'on considère les malades ayant présenté des taux satisfaisants de cyclosporinémie $(>300 \mathrm{ng} / \mathrm{ml}$ à 24 heures) dont $37 \%$ présentaient une rémission complète et $66 \%$ une rémission partielle (moins de to unités d'insuline par jour). Par ailleurs, la fréquence des rémissions se révélait plus élevée chez les malades traités le plus précocément après le début de leur maladie. Aux doses modérées utilisées dans cet essai, la cyclosporine s'est révélée peu toxique. Aucune infection sévère, ni lymphome ne furent observés. La seule complication sérieuse fut une élévation de la créatininémie témoignant d'une néphrotoxicité heureusement totalement réversible à la diminution de la posologie.

Ces résultats $[\mathrm{I}]$ apportent la première démonstration rigoureuse de l'effet préventif du traitement immunosuppresseur sur le cours de la maladie diabétique. Ils valident rétrospectivement les deux essais pilotes antérieurement réalisés au Canada [2] et à Paris [3]. Ils laissent espérer à terme une prévention active de la maladie à mettre en œuvre le plus précocement possible dans l'histoire de la maladie, avant que la majorité des cellules $\beta$ ait été détruite. Il sera néanmoins nécessaire, avant d'en généraliser l'utilisation, de préciser la durée des rémissions ainsi induites et la toxicité à long terme de l'immunosuppression. En raison de ces incertitudes et des problèmes de pharmacocinétique particuliers à la cyclosporine, le nouveau traitement du diabète doit encore être l'objet de protocoles thérapeutiques rigoureux dans des centres spécialisés. 